\title{
Thomas Kühtreiber, Gabriele Schichta (dir.), Kontinuitäten, Umbrüche, Zäsuren
}

Pierre Monnet

\section{OpenEdition}

Journals

Édition électronique

URL : http://journals.openedition.org/ifha/9487

DOI : $10.4000 /$ ifha. 9487

ISSN : 2198-8943

Éditeur

IFRA - Institut franco-allemand (sciences historiques et sociales)

Référence électronique

Pierre Monnet, «Thomas Kühtreiber, Gabriele Schichta (dir.), Kontinuitäten, Umbrüche, Zäsuren », Revue de l'IFHA [En ligne], Date de recension, mis en ligne le 23 août 2018, consulté le 24 septembre 2020 URL : http://journals.openedition.org/ifha/9487 ; DOI : https://doi.org/10.4000/ifha.9487

Ce document a été généré automatiquement le 24 septembre 2020

(CIFHA 


\title{
Thomas Kühtreiber, Gabriele Schichta (dir.), Kontinuitäten, Umbrüche, Zäsuren
}

\author{
Pierre Monnet
}

\section{RÉFÉRENCE}

Thomas Kühtreiber, Gabriele Schichta (dir.), Kontinuitäten, Umbrüche, Zäsuren. Die Konstruktion von Epochen in Mittelalter und früher Neuzeit in interdisziplinärer Sichtung, Heidelberg: Universitätsverlag, 2016, 355 p., $52 €$ 
Ce collectif, issu de rencontres organisées à Krems en 2014, prend acte de deux constats épistémologiques et heuristiques particulièrement prégnants au sein des sciences humaines et sociales depuis plusieurs années: la période, comme toute découpe du temps dans une société, est une construction sociale et idéologique, d'une part; l'organisation et la dénomination des mesures et des effets de temporalité sont propres à la dynamique intellectuelle de chaque discipline, de l'autre. Loin de gêner la réflexion, ces deux observations en forment au contraire le cadre intellectuel : elles constituent une condition et non un obstacle pour tout exercice critique appliqué à la détermination de chronologies et de chrononymes par les sciences de l'homme. Comme le rappelle à

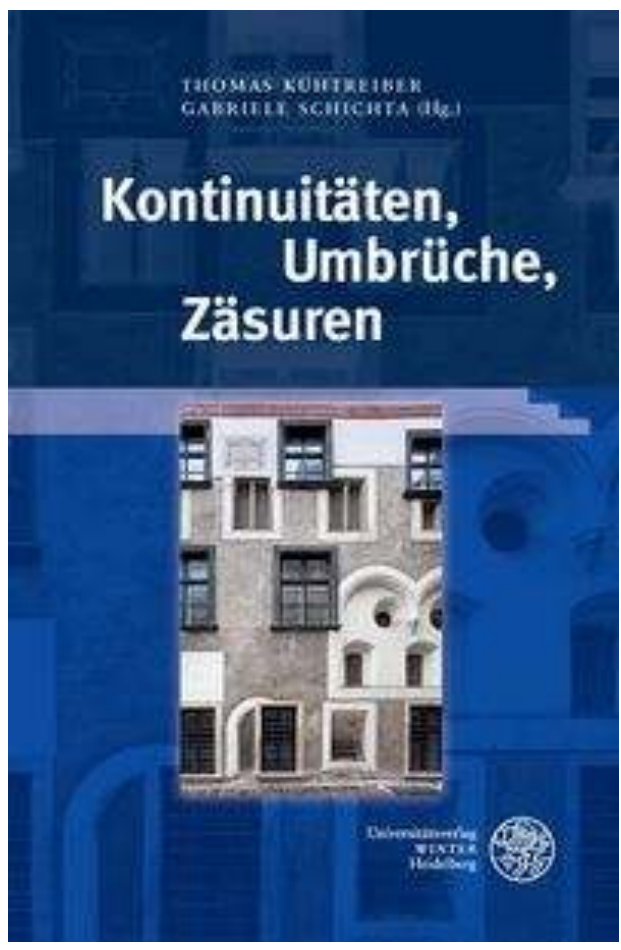
bon droit l'introduction des deux coéditeurs du recueil, toute société cherche à baliser le temps, le sien, mais aussi celui des autres, et celui de son passé, possiblement de son présent et de son futur, selon un régime d'historicité admirablement décortiqué par François Hartog. Qu'il s'agisse là d'un invariant anthropologique (p. 9) peut certes prêter à discussion, mais la remarque vaut en tout cas pour les sociétés fortement historisées d'Occident sur la longue durée de près de trois millénaires (une découpe en soi...).

On peut grossièrement attribuer à au moins quatre phénomènes conjoints (que Gérard Noiriel en son temps avait pu qualifier de «crise » de l'histoire) la remise en cause systématique et impitoyable de la notion d'époque et de période, en histoire comme dans d'autres disciplines et démarches partageant avec elle l'historicité de ses objets, de ses sujets et de ses méthodes : le règne actuellement sans partage du présent et de l'immédiat (et son corrélat que forme l'injonction mémorielle) ; la relativisation voire la négation par l'histoire globale des modèles européens universalisants de pensée historique; l'intégration nécessaire de la propre temporalité de l'historien dans la temporalité de ses interprétations; la prise de conscience de la contemporanéité du non-contemporain.

Dans cette grande épreuve de déconstruction, le Moyen Âge s'est trouvé particulièrement exposé parce que son chrononyme même, transmis de manière assez étonnante jusqu'à nos jours, résultait d'une opération de discrédit lancée par la Renaissance (autre témoin de périodisation...) pour en discréditer les fondements. Peu de périodes académiques traditionnelles ont été à ce point l'objet récent de tortures conceptuelles et temporelles: l'antiquité tardive lui a grignoté en amont plusieurs siècles tandis que la légitime revendication legoffienne d'une longue durée en prolongeait la vie jusqu'aux Lumières en aval. Force est en outre de constater que ces remises en question ou ces déplacements ne se produisent pas semblablement d'une historiographie nationale à l'autre, ainsi que l'Europe actuelle en livre maints 
exemples, et que chaque discipline a opéré ou fixé ses propres glissements à son rythme, entre littérature, musicologie, histoire de l'art, histoire des textes, de l'économie etc. C'est dire si ce volume n'entend pas clore le débat mais plutôt proposer quelques outils de compréhension.

Un premier ensemble de contributions en appelle à un réarmement sémantique et conceptuel: la discussion sur les périodes doit concerner l'ensemble des sciences historiques et sociales, disons sociales en tant qu'elles sont historiques; elle doit s'affronter aux propositions concurrentes (ainsi du remplacement revendiqué de la trilogie Antiquité/Moyen Âge/Temps Modernes par le triptyque Prémoderne/ Moderne/Postmoderne...); elle doit soumettre les opérations de découpes temporelles à la logique de concepts opératoires transversaux (transformation, seuil, mutation, continuité, ancien/neuf, changement paradigmatique, tradition...); elle doit aussi comparer et «mondialiser » les blocs temporels percutés par les notions de culture, de civilisation, d'aire régionale. Un second ensemble d'essais décale un peu le regard en observant comment, dans la période communément dénommée Moyen Âge, des phénomènes non pas semblables mais approchants ont bravé sur le moment les découpes célestes ou terrestres du temps humain en inventant les origines, en confirmant ou en répudiant les légendes, en écrivant la tradition, en se remémorant, en ressuscitant un passé déclaré révolu (l'antique), en fixant des âges d'or, en glosant sur les débuts et la fin du monde et en établissant pour les vivants une manière de traiter leurs morts.

Certes une impression d'hétérogénéité peut se dégager à la lecture successive de 17 contributions alignant dans un ordre plus éditorial que logique l'art, la musique, la médecine, la littérature, l'histoire, l'islam, la peinture, l'encyclopédisme, la scolastique, le blason et la dépouille mortuaire... Toutefois, un fil rouge commun relie le premier ensemble (de nature plus épistémologique) et le deuxième (plus ontologique) : au Moyen Âge comme aujourd'hui la découpe du temps est inséparable d'une activité consistant à tenter de saisir éperdument la complexité et la fugacité du monde, et qui aboutit, sans pour autant s'y épuiser, à l'activité métaphorique, au sens propre du terme grec de metaphora, de transport visant la transposition d'une réalité dans une autre, et sans laquelle il n'existerait pas de langage. À ce titre, hier comme aujourd'hui, l'époque, la période, est bien un effet de langage, un mode et une tentative de compréhension qui n'hésite pas à manier le paradoxe et à transgresser les frontières. En ce sens, l'article le plus percutant et productif du recueil nous semble être celui de Katrin Kärcher et Matthias Heinz (p. 245-263) s'intéressant à la représentation de l'irreprésentable pour un esprit humain fini, à savoir l'éternité. Or, par un retournement saisissant, les deux auteurs montrent bien que c'est en parvenant enfin à maitriser techniquement et allégoriquement "l'irreprésentation » de l'éternité, du non-temps ou du tout-temps, qu'un Francesco da Barberino (1264-1348) signale et exprime un seuil, celui d'un Moyen Âge qui se quitte lui-même en faisant descendre sur terre et en temporalisant l'idée même de la perpétuité. 
INDEX

Thèmes : Histoire des mentalités, Histoire des idées, Historiographie/Méthodologie

\section{AUTEURS}

PIERRE MONNET

EHESS (Paris) et IFRA-SHS (Frankfurt am Main) 